NEW LAGRANGIAN AND HAMILTONIAN METHODS IN FIELD THEORY 
This page is intentionally left blank 


\section{NEW LAGRANGIAN}

\section{AND HAMILTONIAN}

METHODS IN

\section{FIELD THEORY}

\section{G. Giachetta}

Univ. Camerino

L. Mangiarotti

Univ. Camerino

G. Sardanashvily

Moscow State Univ. 


\section{Published by}

World Scientific Publishing Co. Pte. Ltd.

P O Box 128, Farrer Road, Singapore 912805

USA office: Suite 1B, 1060 Main Street, River Edge, NJ 07661

UK office: 57 Shelton Street, Covent Garden, London WC2H 9HE

\section{British Library Cataloguing-in-Publication Data}

A catalogue record for this book is available from the British Library.

\section{NEW LAGRANGIAN AND HAMILTONIAN METHODS IN FIELD THEORY}

Copyright (C) 1997 by World Scientific Publishing Co. Pte. Ltd

All rights reserved. This book, or parts thereof, may not be reproduced in any form or by any means, electronic or mechanical, including photocopying, recording or any information storage and retrieval system now known or to be invented, without written permission from the Publisher.

or photocopying of material in this volume, please pay a copying fee through the Copyright Clearance Center, Inc., 222 Rosewood Drive, Danvers, MA 01923, USA. In this case permission to photocopy is not required from the publisher.

ISBN 981-02-1587-8

This book is printed on acid-free paper.

Printed in Singapore by Uto-Print 


\section{Preface}

This book addresses the application of jet manifold formalism to contemporary classical field theory. This is the natural development of the well-known geometric formulation of field theory, where classical fields are represented by sections of fibred manifolds. In mathematics, the theory of differential operators and the calculus of variations are expressed in terms of jet manifold, which, in field theory, provide the adequate mathematical language for Lagrangian and Hamiltonian formalisms. In general, the book considers only first order Lagrangian and Hamiltonian systems because most contemporary field models are of this type.

Two main peculiarities of the jet formulation of field theory should be emphasized. Firstly, jets of fibred manifolds (when sections are identified by a finite number of terms of their Taylor series) form smooth finite-dimensional manifolds. Therefore, the dynamics of field systems is defined on finite-dimensional configuration and phase spaces. Secondly, jet manifolds provide the language of modern differential geometry to deal with general connections which are represented by sections of jet bundles. As a consequence, the dynamics of field systems include connections in a natural way.

When analytical mechanics is seen as a field theory over a 1-dimensional base, we find a clear illustration of the role of connections. Dynamic, Hamiltonian, and frame connections are the main ingredients in this formulation.

There are two main geometric models of classical field theory which exhaust all observable fundamental fields. These are the gauge theory on principal bundles, including Higgs fields, and the gravitation theory on natural and spinor bundles, including Dirac's fermion matter. In this book, we do not pretend to give a comprehensive description of these models, but to exhibit general methods of investigating classical field systems within the framework of the jet formalism. In the Lagrangian formalism, we use the first variational formula of the calculus of variations as the main tool for discovering the differential conservation laws, including the energymomentum ones, in gauge and gravitation theories. In this connection, spinor fields under deformations of a gravitational field are considered. 
The Hamiltonian counterpart of the Lagrangian formulation of field theory is the covariant polysymplectic Hamiltonian formalism, where canonical momenta correspond to the derivatives of field functions with respect to all world coordinates, not only the temporal one. We investigate the relations between Lagrangian and Hamiltonian formalisms for a wide class of degenerate systems which include almost all contemporary field models. The reduction of the polysymplectic Hamiltonian formalism over a 1-dimensional base provides the adequate mathematical formulation of time-dependent Hamiltonian mechanics in a frame-covariant form.

With respect to mathematical prerequisites, the reader is expected to be familiar with the basics of differential geometry of fibre bundles. In the first two Chapters of the book, we summarize the relevant material on jet manifolds and connections, which is enough for physical applications. At the end of the book, a few topics on jet formalism are reviewed in a more general setting, in order to stimulate further investigations. 


\section{Contents}

Preface v v

Introduction $\quad 1$

1 Fibred Manifolds $\quad 7$

1.1 Immersion and submersion . . . . . . . . . . . . . . . 8

1.2 Fibred manifolds .................... . . 12

1.3 Vector and affine bundles . . . . . . . . . . . . . . 21

1.4 Tangent bundles of fibred manifolds . . . . . . . . . . . . . 25

1.5 Vector and multivector fields . . . . . . . . . . . . . . . 29

1.6 Differential forms on fibred manifolds . . . . . . . . . . . . 33

2 Jet Manifolds and Connections $\quad 43$

2.1 First order jet manifolds . . . . . . . . . . . . . . . . . 43

2.2 Second order jet manifolds . . . . . . . . . . . . . . 52

2.3 Connections . . . . . . . . . . . . . . . . . . 58

2.4 Differentials and codifferentials. Identities ........... 68

2.5 Composite connections . . . . . . . . . . . . . . 75

2.6 Second order connections . . . . . . . . . . . . . . . . . . . 80

2.7 Jets of principal bundles . . . . . . . . . . . . . . 82

2.8 Canonical principal connection . . . . . . . . . . . . . 89

3 Lagrangian Formalism $\quad \mathbf{9 7}$

3.1 Technical preliminaries. Higher order jets . . . . . . . . . . . . . 98

3.2 The first variational formula . . . . . . . . . . . . . . . . 101

3.3 Euler-Lagrange operators . . . . . . . . . . . . . . 108

3.4 Lagrangian polysymplectic structures . . . . . . . . . . . . . . . 121

3.5 Lagrangian conservation laws . . . . . . . . . . . . . . . . . 128

3.6 Conservation laws in gauge theory . . . . . . . . . . . . . . . . . . . . . . . . . . . . . . . . . . .

3.7 Conservation laws in gravitation theory . . . . . . . . . . . 155 
3.8 Gauge gravitation theory . . . . . . . . . . . . . . . . . . 172

3.9 Appendix. Gauge mechanics . . . . . . . . . . . . . . . . . . 204

4 Hamiltonian Formalism 231

4.1 Symplectic structure . . . . . . . . . . . . . . . . 232

4.2 Polysymplectic structure . . . . . . . . . . . . . . . . 241

4.3 Hamiltonian forms . . . . . . . . . . . . . . . . . 247

4.4 Hamilton equations . . . . . . . . . . . . . . . . . . . . 252

4.5 Degenerate systems . . . . . . . . . . . . . . . . . . 261

4.6 Quadratic degenerate systems ... . . . . . . . . . . . . 281

4.7 Affine degenerate systems . . . . . . . . . . . . . . . . 296

4.8 Hamiltonian conservation laws . . . . . . . . . . . . . . . . . 301

4.9 Vertical extension of polysymplectic formalism . . . . . . . . . 305

4.10 Appendix. Hamiltonian time-dependent mechanics . . . . . . . . 308

5 Special Topics $\mathbf{3 3 3}$

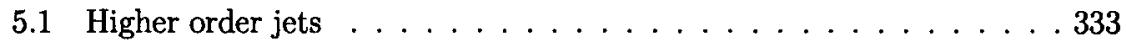

5.2 Jets of modules . . . . . . . . . . . . . . . . . . . . . . . . . . . . . . . . . . . . .

5.3 Jets of submanifolds . . . . . . . . . . . . . . . 362

5.4 Infinite order jets . . . . . . . . . . . . . . . . . . . . . . . . . . . . . . . .

5.5 Variational bicomplex . . . . . . . . . . . . . . . . . . . . . . . . . . . . . . . . . .

5.6 Geometry of differential equations . . . . . . . . . . . . . . 386

5.7 Formal integrability . . . . . . . . . . . . . . . 405

$\begin{array}{lr}\text { Bibliography } & 427\end{array}$

Glossary of Symbols $\quad 443$

$\begin{array}{lr}\text { Index } & 449\end{array}$ 